\title{
Clinical symptoms and psychological changes of patients with COVID-19 in Jiangxi Province
}

\section{Jing Zhou}

First Affiliated Hospital of Nanchang University

\section{Xin-Ping Xu}

First Affiliated Hospital of Nanchang University

\section{Fei Xu}

First Affiliated Hospital of Nanchang University

\section{Yi Shao}

The first affiliated hospital of Nanchang University

\section{Mei-Hong Zou}

The first affiliated hospital of Nanchang University

\section{Jing-Jing Yu}

First Affiliated Hospital of Nanchang University

\section{Fen Liu}

First Affiliated Hospital of Nanchang University

\section{Wei Zuo}

First Affiliated Hospital of Nanchang University

\section{Si-Guang Xie}

First Affiliated Hospital of Nanchang University

\section{Cong-Yang Zhou}

First Affiliated Hospital of Nanchang University

\section{Wei Zhang ( $\sim$ zhangweiliuxin@163.com )}

First Affiliated Hospital of Nanchang University https://orcid.org/0000-0002-3716-1831

\section{Research}

Keywords: Corona virus disease 2019 (COVID-19), novel coronavirus pneumonia (SARS-COV-2), psychological changes, the Hospital Anxiety and Depression Scale (HADS), the Mini-Mental State Examination scale, Van Dream Anxiety Scale score

Posted Date: March 19th, 2020

DOI: https://doi.org/10.21203/rs.3.rs-18080/v1 
License: (c) (i) This work is licensed under a Creative Commons Attribution 4.0 International License. Read Full License 


\section{Abstract}

Objective

The purpose of this study was to determine the prevalence and differences in etiology, clinical manifestations, and psychological activity of coronavirus disease-19 (COVID-19) among patients.

Results

We recruited 90 subjects, 30 were healthy controls, 30 were patients with moderate infection, and 30 were patients with severe/critical infections. No significant differences were noted in the sex ratio, mean age, body mass index, or blood type; however, the history of exposure of the patients with COVID-19 compared with healthy controls was noteworthy. The erythrocyte sedimentation rate, as well as the levels of C-reactive protein and serum amyloid A (SAA) were all increased. In terms of mental health, there were significant differences in the worry scores between severely and moderately infected patients and healthy controls. There was a significant difference in depression scores between patients with moderate infection and healthy hypertension, and there was also a significant difference in dream worry scores. Analysis of the Mini-Mental State Examination scores showed that for patients with moderate infection, the depression score was moderately and positively correlated with the dream anxiety score. For patients with severe infection, the anxiety score was positively correlated with the dream anxiety score, and the depression score was moderately and positively correlated with the dream anxiety score.

Conclusion

Patients with severe infection showed increased pain and sputum in the pharyngeal area compared with patients with moderate infection. Patients with blood type A may be more susceptible to COVID-19, and lymphopenia may indicate worsening of COVID-19.

\section{Introduction}

Since December 2019, the outbreak of a new coronavirus disease (COVID-19) has become a major epidemic threat in China. As of March 10, 2020, there have been 80,778 confirmed cases in mainland China, 61,475 cured cases $(76.10 \%)$, and 3,158 deaths $(3.91 \%) .{ }^{1}$ All 31 provinces in mainland China have adopted first-level responses to major public health emergencies. The central government and some provincial governments have provided food and medicine, and have sent expert and medical teams to manage and control the most affected areas (outbreak response in Wuhan and surrounding cities in Hubei Province). On February 24, 2020, Chinese members of parliament began to consider a draft decision to completely ban the illegal wildlife trade and eliminate the undesirable habit of the consumption of wild animals to protect public health. ${ }^{1}$

On January 12, 2020, the World Health Organization (WHO) temporarily named the new virus "2019-New Coronavirus (2019-nCoV)." ${ }^{2}$ Subsequent research confirmed that 2019-nCoV belongs to a new type of $\beta$ coronavirus, because its genetic characteristics differed significantly from those of the severe acute 
respiratory syndrome coronavirus (SARS-CoV) and Middle Eastern respiratory syndrome coronavirus (MERS-CoV). Furthermore, its homology with bat-sl-covzc45 exceeded $85 \%{ }^{3}{ }^{3}$ thus, the WHO renamed it to the "severe acute respiratory syndrome coronavirus 2 (SARS-CoV-2)." ${ }^{4}$ Prior to this, six coronaviruses were known to cause human disease, including alpha 229E and NL63, HKU1, OC43, SARS-CoV, and beta MERSCoV. In normal individuals, 229E, NL63, HKU1, and OC43 only causes common cold symptoms, while severe SARS-CoV and MERS-CoV can cause death. ${ }^{5.6}$ On February 13,2020 , the WHO officially named pneumonia caused by SARS-CoV-2 "COVID-19."

The National Health Commission of the People's Republic of China reported on February 6, 2020 in the Handbook of Prevention and Treatment of the Pneumonia Caused by the Novel Coronavirus (2019-nCoV), details of the currently known pathogenic characteristics of the new coronavirus, and introduced some practicality in conjunction with the current situation. ${ }^{7}$

The new coronavirus is highly contagious and can be transmitted from person to person through respiratory droplets or contact. The general population is susceptible. Epidemiological investigation shows that the incubation period of the new coronavirus pneumonia is generally $3-7$ days, and the longest incubation does not exceed 14 days. ${ }^{8}$ It differs from SRAS-CoV, as COVID-19 is also contagious during the incubation period. ${ }^{9}$ In addition, interpersonal transmission can occur both in hospitals and in the home environment. ${ }^{10}$ COVID-19 outbreaks have occurred in many cities in China, and have expanded globally, including South Korea and Japan, among other countries. This has led the WHO to announce a Public Health Emergency of International Concern (PHEIC). ${ }^{11}$

After analyzing the diagnosis and treatment experience of cured patients, we concluded that early identification, reporting, isolation, diagnosis, and treatment constitute some of the best and most effective ways to curb pneumonia caused by SARS-CoV-2. ${ }^{12}$ At the same time, the efficacy of traditional Chinese and western medicine has been recognized. Nationally, more than $75 \%$ of patients with COVID-19 in Hubei have been receiving traditional Chinese medicine treatment, compared with more than $90 \%$ in other parts of China. ${ }^{12}$

Thus far, despite new coronavirus outbreaks, China has launched public health emergency interventions for mental health, such as the establishment of a mental health hotline and the establishment of nationwide telephone and internet-based counseling services to facilitate patient knowledge, alleviate fears, and solve problems related to mental health. ${ }^{12}$ The National Health Commission has even issued guidelines for local authorities to promote psychological crisis interventions for patients, medical staff, and people under medical observation. ${ }^{13}$ However, the type of influence the patient's mental health status or mental state may have on the course of the disease, and the extent to which it may be affected remains unknown. To our knowledge, no scientific study has been conducted to analyze the relationship between the mental health of the patient and the course of the disease.

Therefore, we aimed to use the Hospital Anxiety and Depression Scale (HADS), Mini-Mental State Examination (MMSE), and Van Dream Anxiety Scales, which are common clinical assessments to 
determine anxiety, depression, and the mental state. We also predict and analyze health status, mental health status of patients with moderate and severe infections, to understand the correlation between mental status and disease severity. In addition, we performed statistical analysis of the epidemiological data, clinical signs, as well as the imaging and laboratory test results of patients to provide timely frontline information.

\section{Materials And Methods}

\subsection{Subjects and study design}

A total of 60 patients with COVID-19 were admitted to the First Affiliated Hospital of Nanchang University from January 27 to February 10, 2020, including 30 patients with moderate infection and 30 patients with severe infection (21 patients with severe infection and nine critical patients). We collected epidemiological and clinical data of the patients after admission, and performed laboratory tests, such as routine blood tests, and those to determine erythrocyte sedimentation, C-reactive protein, calcitonin, myocardial enzymes, liver and kidney function, electrolytes, coagulation function, $\mathrm{T}$ cell subsets, and immune indicators, among others. Chest computed tomography (CT) scans were also acquired.

According to the clinical typing method of the "New Coronavirus Infected Pneumonia Diagnosis and Treatment Scheme (Trial Version 4)," the clinical characteristics of patients with moderate infection and those with severe/critical infections were compared and analyzed. We then randomly recruited 30 healthy controls. The age and sex of the healthy controls were consistent with those of both the patients with moderate infection and those with severe infections. The exclusion criteria were as follows: any chronic underlying diseases such as hypertension, diabetes, hepatitis B, etc.

All subjects and their families voluntarily signed informed consent forms. We asked a total of 90 subjects to complete three scales (the HADS, MMSE, and the Van Dream Anxiety Scale), and compared and analyzed the scores of each among the three groups. We were able to determine the mental health status of patients with moderate infection and those with severe infection, and identify the relationship between mental health and severity of the disease.

This was a prospective intervention study based on the Helsinki Declaration that had been approved by the Ethics Committee of the First Affiliated Hospital of Nanchang University. All methods were performed to the extent permitted by relevant guidelines and regulations. The inclusion criteria for patients with COVID-19 met any of the following conditions: (1) real-time fluorescent reverse transcription polymerase chain reaction (RT-PCR) detection of new coronavirus nucleic acids isolated from respiratory or blood samples; (2) comparison with known new coronaviruses showed highly homologous viral gene sequences from respiratory or blood samples.

All experimental procedures were repeated more than twice, with an interval of more than $24 \mathrm{~h}$. Patients with severe infection met any of the following conditions: (1) respiratory distress, breathing frequency $\geq 30$ times/minute; (2) in a resting state, oxygen saturation $\leq 93 \%$; (3) partial arterial pressure of oxygen $\left(\mathrm{PaO}_{2}\right)$ 
or the concentration of oxygen $\left(\mathrm{FiO}_{2}\right) \leq 300 \mathrm{mmHg}(1 \mathrm{mmHg}=0.133 \mathrm{kPa})$. In addition, critically ill patients met any of the following conditions: (1) respiratory failure requiring mechanical ventilation; (2) shock; (3) other organ failure requiring intensive care unit (ICU) monitoring and treatment.

\subsection{Pharmacological agents}

Clinical treatment strategies for patients with COVID-19 were based on previous experience with SARS-CoV and MERS-CoV. For patients with moderate infection, we administered moxifloxacin (30 of 30 patients [100.00\%]) and lopinavir ritonavir tablets (26 of 30 patients [86.67\%]), arbidol hydrochloride (2 of 30 patients [6.67\%]), or combination therapy with lopinavir ritonavir tablets and immunoglobulins (2 of 30 patients [6.67\%]). For patients with severe infection, we administered moxifloxacin (30 of 30 patients [100.0\%]) and lopinavir ritonavir tablets (26 of 30 patients [86.67\%]) in combination. At the same time, we added immunoglobulin (8 of 30 patients [26.67\%]), methylprednisolone (8 of 30 patients [26.67\%]), arbidol hydrochloride (5 of 30 patients [16.67\%]), cefoperazone sodium and sulbactam sodium (5 of 30 patients [16.67\%]), interferon ( 2 of 30 patients [6.67\%]), ceftriaxone (1 of 30 patients [3.33\%]), thymosin (1 of 30 patients [3.33\%] ), and oseltamivir phosphate (1 of 30 patients [3.33\%]).

\subsection{Data collection}

Prior to participating in the study, all subjects or their families voluntarily gave written informed consent. Through adequate medical history taking and detailed physical examination, we obtained the age and weight of 60 patients with COVID-19, admitted to the First Affiliated Hospital of Nanchang University from January 27 to February 10, 2020. We also recruited 30 healthy controls.

The body mass index (BMI), contact history, incubation period, temperature, pulse, respiratory rate, systolic blood pressure, diastolic blood pressure, and other clinical data were recorded. Laboratory tests, including routine blood tests, as well as those to determine erythrocyte sedimentation, C-reactive protein, calcitonin, myocardial enzymes, liver and kidney function, electrolytes, coagulation function, and $\mathrm{T}$ cell subsets were performed, and chest CT scans were obtained. According to the clinical classification method of "New Coronavirus Infected Pneumonia Diagnosis and Treatment Scheme (Trial Version 4)," 60 patients were divided into two groups; 30 patients with moderate infection and 30 patients with severe infection (including 21 patients with severe infection and nine critical patients).

We asked all 90 subjects to complete three scales (the HADS, MMSE scale, and the Van Dream Anxiety Scale). The HADS self-assessment scale is a reliable tool used for the detection of depression and anxiety in hospital outpatients. Thus, it can provide data on the severity of anxiety and depression. The MMSE scale is easy to implement and is widely used locally and abroad. It includes the following elements: timeoriented power, position-oriented power, instant memory, attention and computing power, delayed memory, language, and visual space. The Van Dream Anxiety Scale, as a subjective measure of anxiety during nightmares, can re-verify the true degree of anxiety to a certain extent.

The above three scales were applicable for the assessment of the psychological status and mental state of patients with COVID-19. By comparing and analyzing the HADS, MMSE, and Van Dream Anxiety Scale 
scores of the three groups of subjects, we were able to determine their mental health status, and identify any relationships between mental health and severity of the disease.

\subsection{Statistical analyses}

One-way analysis of variance (one-way ANOVA) in the SPSS ${ }^{8}$ (SPSS Inc., Chicago, IL, USA) statistical analysis computer package version 17.0 was used to analyze the basic clinical characteristics (including sex, age, and BMI) of patients with moderate infection and those with severe infection, as well as the scale scores (such as anxiety score and depression score).

In addition, the chi-squared test and t-test were used to evaluate clinical symptoms and physical examination data, such as the incubation period, course of disease, pulmonary CT lesions, and body temperature in patients with moderate infection and those with severe infection. Further analysis was conducted on the results of laboratory tests of immune indicators, including routine blood tests, tests of erythrocyte sedimentation, C-reactive protein, calcitonin, myocardial enzymes, liver and kidney function, electrolytes, coagulation function, and T cell subsets in the patients with moderate infection and those with severe infection to determine the any differences between the two groups. A P-value of $<0.05$ was considered statistically significant.

\section{Results}

\subsection{Comparison of chest CT images between healthy controls and patients with COVID-19}

We found no lung lesions in the healthy controls, while the number of lung lesions in patients with COVID19 was $2.68 \pm 1.61$. The CT images of the lungs in healthy controls and in patients with COVID-19 are shown in Fig. 1.

[Figure 1]

\subsection{Clinical characteristics, symptoms, and radiographic findings}

A total of 90 study subjects were considered for analysis, including 30 healthy controls, 30 patients with moderate infection, and 30 patients with severe infection (including 21 patients with severe infection and nine critical patients). No significant differences were noted in sex ratio, mean age, BMI, or blood type (all $\mathrm{P}$ $>0.05)$. However, the history of exposure among the patients with COVID-19 compared with that of the healthy controls was noteworthy $(P<0.001)$.

Among both the patients with moderate infection and those with severe infection, the proportion of patients with blood type $A$ in confirmed cases was higher. Patients with moderate infection and those with severe infection showed no significant differences in the incubation period $(P=0.577)$, disease period $(P=0.481)$, body temperature $(P=0.720)$, pulse $(P=0.658)$, respiration $(P=0.286)$, systolic blood pressure $(P=0.704)$, diastolic blood pressure $(P=0.564)$, or initial symptoms $(P=0.257)$. However, the chest $C T$ images were still 
able to facilitate comparisons among the lungs of ordinary patients, and those with moderate and severe infections.

Interstitial abnormalities accounted for the largest proportion of clinical signs observed in patients with severe infection (23.33\%). Significant differences were noted in symptoms on admission between the patients with moderate infection and those with severe infection $(P=0.002)$. In patients with moderate infection, the symptoms present on admission were mainly fever (43.33\%) and cough (26.67\%), while patients with severe infection had fever $(80.00 \%)$, cough (43.33\%), sputum $(20.00 \%)$, and sore throat (20.00\%), among other symptoms.

Furthermore, seven patients $(11.67 \%)$ had complications related to chronic underlying diseases, such as hypertension, diabetes, and hepatitis B. Tables 1 and 2 show the detailed epidemiological data and clinical symptoms of healthy controls, patients with moderate infection, and patients with severe infection. In addition, Fig. 2 shows the distribution of patients with severe infection among the study participants. 
Table 1

Demographic, psychological and imaging characteristics of COVID-19 patients

\begin{tabular}{|c|c|c|c|c|c|c|c|}
\hline & $\begin{array}{l}\text { Healthy } \\
\text { control }(n=30)\end{array}$ & $\begin{array}{l}\text { Common } \\
\text { patients }(\mathrm{n}= \\
30)\end{array}$ & $\begin{array}{l}\text { Severe } \\
\text { patients }(\mathrm{n}= \\
30)\end{array}$ & $P_{1}$ & $P_{2}$ & $P_{3}$ & $\mathrm{P}_{4}$ \\
\hline $\begin{array}{l}\text { Sex-No./total } \\
\text { No. (\%) }\end{array}$ & & & & 0.483 & 0.292 & 1.000 & 0.292 \\
\hline male & 16/30(53.33) & $20 / 30(66.67)$ & 16/30(53.33) & - & - & - & - \\
\hline female & $14 / 30(46.67)$ & 10/30(33.33) & $14 / 30(46.67)$ & - & - & - & - \\
\hline Age (years) & $39.30 \pm 13.57$ & $\begin{array}{l}45.50 \pm \\
13.88\end{array}$ & $\begin{array}{l}39.87 \pm \\
14.78\end{array}$ & 0.175 & 0.092 & 0.877 & 0.125 \\
\hline Anxiety score & $3.63 \pm 1.43$ & $4.73 \pm 3.13$ & $6.60 \pm 2.54$ & $<.001$ & 0.088 & $\begin{array}{l}< \\
0.001\end{array}$ & 0.004 \\
\hline $\begin{array}{l}\text { Depression } \\
\text { score }\end{array}$ & $3.60 \pm 1.61$ & $4.77 \pm 3.10$ & $6.13 \pm 3.09$ & 0.002 & 0.097 & $<.001$ & 0.053 \\
\hline $\begin{array}{l}\text { MMSE scale } \\
\text { score }\end{array}$ & $31.57 \pm 3.08$ & $25.83 \pm 3.17$ & $27.60 \pm 2.70$ & $<.001$ & $\begin{array}{l}< \\
0.001\end{array}$ & $\begin{array}{l}< \\
0.001\end{array}$ & 0.025 \\
\hline $\begin{array}{l}\text { Dream anxiety } \\
\text { score }\end{array}$ & $17.93 \pm 5.06$ & $\begin{array}{l}28.90 \pm \\
11.58\end{array}$ & $\begin{array}{l}35.07 \pm \\
12.60\end{array}$ & $<.001$ & $<.001$ & $<$. & 0.023 \\
\hline BMI (kg / m2) & $18.87 \pm 1.73$ & $19.20 \pm 1.71$ & $19.92 \pm 2.53$ & 0.132 & 0.533 & 0.050 & 0.176 \\
\hline $\begin{array}{l}\text { Blood group- } \\
\text { No./total No. } \\
(\%)\end{array}$ & & & & 0.626 & 0.433 & 0.983 & 0.270 \\
\hline A & $15 / 30(50.00)$ & $15 / 30(50.00)$ & $15 / 30(50.00)$ & - & - & - & - \\
\hline B & $5 / 30(13.33)$ & $9 / 30(30.00)$ & $4 / 30(13.33)$ & - & - & - & - \\
\hline
\end{tabular}

Notes: One-Way analysis of variance (One-Way ANOVA), Chi-squared tests or the two-tailed, paired Student's t-test were used. A P-value $<0.05$ was considered statistically significant. P1 represents the P value between three groups; $\mathrm{P} 2$ represents the $\mathrm{P}$ value of healthy controls and ordinary patients; P3 represents the $P$ value of normal patients and severe patients; $P 4$ represents the $P$ value of ordinary patients and severe patients. Abbreviations: BMI, body mass index; MMSE, Mini-Mental State Examination. 


\begin{tabular}{|c|c|c|c|c|c|c|c|}
\hline & $\begin{array}{l}\text { Healthy } \\
\text { control }(n=30)\end{array}$ & $\begin{array}{l}\text { Common } \\
\text { patients }(n= \\
\text { 30) }\end{array}$ & $\begin{array}{l}\text { Severe } \\
\text { patients }(n= \\
30)\end{array}$ & $P_{1}$ & $P_{2}$ & $P_{3}$ & $P_{4}$ \\
\hline 0 & 9/30(33.33) & $6 / 30(20.00)$ & 10/30(33.33) & - & - & - & - \\
\hline$A B$ & 1/30(3.33) & $0 / 30(0.00)$ & 1/30(3.33) & - & - & - & - \\
\hline $\begin{array}{l}\text { Contact } \\
\text { history- } \\
\text { No./total No. } \\
(\%)\end{array}$ & & & & $<.001$ & $\begin{array}{l}< \\
0.001\end{array}$ & $\begin{array}{l}<.001 \\
0.00\end{array}$ & 0.688 \\
\hline Yes & $0 / 30(0.00)$ & $27 / 30(90.00)$ & $26 / 30(86.67)$ & - & - & - & - \\
\hline No & $30 / 30(100.00)$ & $3 / 30(10.00)$ & 4/30(13.33) & - & - & - & - \\
\hline $\begin{array}{l}\text { Incubation } \\
\text { period(day) }\end{array}$ & - & $5.30 \pm 4.02$ & $5.40 \pm 4.77$ & - & - & - & 0.577 \\
\hline $\begin{array}{l}\text { Duration of } \\
\text { symptoms(day) }\end{array}$ & - & $5.13 \pm 3.82$ & $5.53 \pm 2.54$ & - & - & - & 0.481 \\
\hline $\begin{array}{l}\text { Abnormalities } \\
\text { on chest CT }\end{array}$ & & & & - & - & - & 0.034 \\
\hline $\begin{array}{l}\text { Local ground- } \\
\text { glass opacity }\end{array}$ & - & $0 / 30(0.00)$ & 1/30(3.33) & - & - & - & - \\
\hline $\begin{array}{l}\text { Bilateral } \\
\text { ground-glass } \\
\text { opacity }\end{array}$ & - & 4/30(13.33) & $5 / 30(16.67)$ & - & - & - & - \\
\hline $\begin{array}{l}\text { Local patchy } \\
\text { shadowing }\end{array}$ & - & $0 / 30(0.00)$ & 3/30(10.00) & - & - & - & - \\
\hline $\begin{array}{l}\text { Bilateral patchy } \\
\text { shadowing }\end{array}$ & - & 8/30(26.67) & 6/30(20.00) & - & - & - & - \\
\hline $\begin{array}{l}\text { Bilateral } \\
\text { multiple } \\
\text { exudative } \\
\text { lesions }\end{array}$ & - & $3 / 30(10.00)$ & 4/30(13.33) & - & - & - & - \\
\hline $\begin{array}{l}\text { Interstitial } \\
\text { abnormalities }\end{array}$ & - & $2 / 30(6.67)$ & 7/30(23.33) & - & - & - & - \\
\hline Non & - & $15 / 30(50.00)$ & 4/30(13.33) & - & - & - & - \\
\hline \multicolumn{8}{|c|}{$\begin{array}{l}\text { Notes: One-Way analysis of variance (One-Way ANOVA), Chi-squared tests or the two-tailed, paired } \\
\text { Student's t-test were used. A P-value < } 0.05 \text { was considered statistically significant. P1 represents the P } \\
\text { value between three groups; P2 represents the P value of healthy controls and ordinary patients; P3 } \\
\text { represents the P value of normal patients and severe patients; P4 represents the P value of ordinary } \\
\text { patients and severe patients. Abbreviations: BMI, body mass index; MMSE, Mini-Mental State } \\
\text { Examination. }\end{array}$} \\
\hline
\end{tabular}


Table 2

Basic characteristics and clinical symptoms of patients with COVID-19

\begin{tabular}{|c|c|c|c|}
\hline Basic characteristics & $\begin{array}{l}\text { Common patients }(\mathrm{n}= \\
\text { 30) }\end{array}$ & $\begin{array}{l}\text { Severe patients }(n= \\
\text { 30) }\end{array}$ & $\mathbf{P}$ \\
\hline $\mathrm{T}\left({ }^{\circ} \mathrm{C}\right)$ & $37.12 \pm 0.68$ & $37.19 \pm 0.75$ & 0.720 \\
\hline $\mathrm{P}$, per minute & $93.43 \pm 9.49$ & $92.20 \pm 11.86$ & 0.658 \\
\hline $\mathrm{R}$, per minute & $20.27 \pm 2.60$ & $19.67 \pm 1.60$ & 0.286 \\
\hline \multicolumn{4}{|l|}{ BP } \\
\hline $\mathrm{SDP}(\mathrm{mmHg})$ & $126.50 \pm 16.20$ & $124.93 \pm 15.53$ & 0.704 \\
\hline DBP (mmHg) & $84.70 \pm 11.35$ & $82.73 \pm 14.71$ & 0.564 \\
\hline First symptoms-No./total No. (\%) & & & 0.257 \\
\hline Fever & $24 / 30(80.00)$ & $27 / 30(90.00)$ & - \\
\hline Cough & 10/30(33.33) & $15 / 30(50.00)$ & - \\
\hline Sputum production & $2 / 30(6.67)$ & $7 / 30(23.33)$ & - \\
\hline Sore throat & $4 / 30(13.33)$ & $9 / 30(30.00)$ & - \\
\hline Fatigue & $8 / 30(26.67)$ & $5 / 30(16.67)$ & - \\
\hline Chill & $4 / 30(13.33)$ & $2 / 30(6.67)$ & - \\
\hline Nasal congestion & $0 / 30(0.00)$ & $1 / 30(3.33)$ & - \\
\hline Runny nose & $0 / 30(0.00)$ & $1 / 30(3.33)$ & - \\
\hline Headache & $4 / 30(13.33)$ & $1 / 30(3.33)$ & - \\
\hline Nausea or vomiting & $2 / 30(6.67)$ & $1 / 30(3.33)$ & - \\
\hline Dyspnoea or chest tightness & $1 / 30(3.33)$ & $3 / 30(10.00)$ & - \\
\hline Mylgia or arthralgia & $1 / 30(3.33)$ & $1 / 30(3.33)$ & - \\
\hline Diarrhea & $3 / 30(10.00)$ & $0 / 30(0.00)$ & - \\
\hline $\begin{array}{l}\text { Admission symptoms-No./total No. } \\
(\%)\end{array}$ & & & 0.002 \\
\hline Fever & $13 / 30(43.33)$ & $24 / 30(80.00)$ & - \\
\hline Cough & $8 / 30(26.67)$ & $13 / 30(43.33)$ & - \\
\hline Sputum production & $4 / 30(13.33)$ & $6 / 30(20.00)$ & - \\
\hline
\end{tabular}




\begin{tabular}{|c|c|c|c|}
\hline Basic characteristics & $\begin{array}{l}\text { Common patients }(\mathrm{n}= \\
\text { 30) }\end{array}$ & $\begin{array}{l}\text { Severe patients }(n= \\
\text { 30) }\end{array}$ & $\mathbf{P}$ \\
\hline Sore throat & $0 / 30(0.00)$ & $6 / 30(20.00)$ & - \\
\hline Fatigue & $3 / 30(10.00)$ & $5 / 30(16.67)$ & - \\
\hline Chill & $1 / 30(3.33)$ & $1 / 30(3.33)$ & - \\
\hline Nasal congestion & $0 / 30(0.00)$ & $0 / 30(0.00)$ & - \\
\hline Runny nose & $1 / 30(3.33)$ & $0 / 30(0.00)$ & - \\
\hline Headache or dizziness & $4 / 30(13.33)$ & $3 / 30(10.00)$ & - \\
\hline Nausea or vomiting & $0 / 30(0.00)$ & $1 / 30(3.33)$ & - \\
\hline Dyspnoea or chest tightness & $2 / 30(6.67)$ & $4 / 30(13.33)$ & - \\
\hline Mylgia or arthralgia & $0 / 30(0.00)$ & $2 / 30(6.67)$ & - \\
\hline Diarrhea & $0 / 30(0.00)$ & $0 / 30(0.00)$ & - \\
\hline Palpitations & $2 / 30(6.67)$ & $0 / 30(0.00)$ & - \\
\hline Non & $14 / 30(46.67)$ & $0 / 30(0.00)$ & - \\
\hline $\begin{array}{l}\text { Notes: Chi-squared tests or th } \\
\text { considered statistically signi } \\
\text { BP, blood pressure; SBP, syst }\end{array}$ & $\begin{array}{l}\text { aired Student's t-test v } \\
\text { ations: T, temperature; } \\
\text { sure; DBP, diastolic blc }\end{array}$ & $\begin{array}{l}\text { sed. A P-value }<0.05 \\
\text { se frequency; R, resp } \\
\text { ssure. }\end{array}$ & \\
\hline
\end{tabular}

\subsection{Laboratory findings}

Serum lymphocyte counts and serum calcium were lower in the admitted patients, while T lymphocyte subsets $(\mathrm{CD} 3+, \mathrm{CD} 4+, \mathrm{CD} 8+)$, B lymphocyte subsets (CD19 +), and natural killer cells (CD56 +, CD16 +) were all significantly decreased. The erythrocyte sedimentation rate, C-reactive protein, and serum amyloid A (SAA) were all increased. Compared with the patients with moderate infection, aspartate aminotransferase (AST) levels were lower in patients with severe infection $(P=0.250)$ and $D$-dimer levels were increased $(P=0.163)$. Compared with the patients with severe infection, low-density lipoprotein $(L D L)$ $(P=0.343)$, fibrin $(P=0.086)$, and serum potassium were higher in patients with moderate infection $(P=$ 0.329). Table 3 details the results of laboratory tests. 
Table 3

Blood routine, liver and kidney function and immunological characteristics of patients with COVID-19 at admission

\begin{tabular}{|c|c|c|c|}
\hline & Common patients $(n=30)$ & Severe patients $(n=30)$ & $\mathbf{P}$ \\
\hline WBC, $\times 10 \rrbracket / L$ & $5.08 \pm 2.32$ & $5.03 \pm 3.44$ & 0.944 \\
\hline $\mathrm{RBC}, \times 10^{12} / \mathrm{L}$ & $4.73 \pm 0.49$ & $4.42 \pm 0.52$ & 0.023 \\
\hline $\mathrm{HGB}, \mathrm{g} / \mathrm{L}$ & $146.90 \pm 17.82$ & $140.47 \pm 16.11$ & 0.148 \\
\hline $\mathrm{ESR}, \mathrm{mm} / \mathrm{h}$ & $42.23 \pm 26.95$ & $44.60 \pm 21.35$ & 0.708 \\
\hline HCT, \% & $0.42 \pm 0.05$ & $0.41 \pm 0.04$ & 0.113 \\
\hline 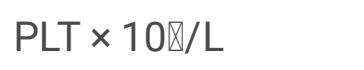 & $170.60 \pm 77.27$ & $155.43 \pm 52.43$ & 0.377 \\
\hline$L Y M P, \times 10 \rrbracket / L$ & $1.31 \pm 0.71$ & $1.13 \pm 0.47$ & 0.236 \\
\hline MON\#, × 10ه/L & $0.41 \pm 0.20$ & $0.35 \pm 0.14$ & 0.202 \\
\hline NEUT $\times 10 \otimes / L$ & $3.32 \pm 1.65$ & $3.33 \pm 2.29$ & 0.978 \\
\hline 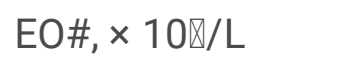 & $0.05 \pm 0.10$ & $0.22 \pm 1.14$ & 0.411 \\
\hline CPR, mg/L & $24.16 \pm 32.49$ & $17.18 \pm 20.39$ & 0.323 \\
\hline SAA, mg/L & $84.36 \pm 80.92$ & $92.06 \pm 94.68$ & 0.736 \\
\hline $\mathrm{CT}, \mathrm{ng} / \mathrm{L}$ & $0.05 \pm 0.03$ & $0.05 \pm 0.09$ & 0.833 \\
\hline Albumin, $g / L$ & $43.86 \pm 3.68$ & $43.92 \pm 4.46$ & 0.954 \\
\hline Globulin, g/L & $26.37 \pm 3.35$ & $28.03 \pm 5.24$ & 0.151 \\
\hline $\mathrm{TB}, \mu \mathrm{mol} / \mathrm{L}$ & $7.67 \pm 2.80$ & $8.16 \pm 3.99$ & 0.582 \\
\hline D-BIL, $\mu \mathrm{mol} / \mathrm{L}$ & $2.74 \pm 1.07$ & $2.58 \pm 1.24$ & 0.602 \\
\hline ALT, U/L & $26.63 \pm 26.73$ & $20.47 \pm 13.00$ & 0.261 \\
\hline AST, U/L & $33.73 \pm 40.69$ & $24.83 \pm 10.07$ & 0.250 \\
\hline AKP, u/l & $68.83 \pm 27.03$ & $67.40 \pm 26.44$ & 0.836 \\
\hline Creatinine, $\mu \mathrm{mol} / \mathrm{L}$ & $66.68 \pm 15.41$ & $64.51 \pm 16.43$ & 0.599 \\
\hline
\end{tabular}

Abbreviations: WBC, white blood cell count; RBC, red blood cell count; HGB, hemoglobin; ESR, erythrocyte sedimentation rate; $\mathrm{HCT}$, hematocrit; PLT, Platelet count; LYMP, lymphocyte count; MON\#, monocytes absolute count; NEUT, neutrophil count; EO\#, eosinophil absolute count; CRP, C-reactive protein; SAA, serum amyloid A; CT, calcitonin; TB, Total bilirubin; D-BIL, direct bilirubin; ALT, alanine aminotransferase; AST, aspartate aminotransferase; AKP, alkaline phosphatase; LDH, Lactate dehydrogenase; CK, creatine kinase; TG, triglyceride; TC, total cholesterol; HDL, high-density lipoprotein; LDL, low-density lipoprotein; PT, prothrombin time; D-D, D dimer; APTT, activated partial thromboplastin time; INR, international normalized ratio; CD, cluster differentiation antigen; C, complement. 


\begin{tabular}{|c|c|c|c|}
\hline & Common patients $(n=30)$ & Severe patients $(n=30)$ & $\mathbf{P}$ \\
\hline Urea, mmol/l & $4.22 \pm 1.24$ & $4.39 \pm 1.60$ & 0.661 \\
\hline Uric acid, $\mu \mathrm{mol} / \mathrm{L}$ & $255.13 \pm 78.99$ & $257.83 \pm 64.60$ & 0.885 \\
\hline LDH, U/L & $256.93 \pm 104.62$ & $253.07 \pm 130.48$ & 0.901 \\
\hline $\mathrm{CK}, \mathrm{U} / \mathrm{L}$ & $134.20 \pm 143.58$ & $125.43 \pm 97.45$ & 0.783 \\
\hline creatine kinase isoenzyme, $\mathrm{U} / \mathrm{L}$ & $12.20 \pm 4.23$ & $15.57 \pm 8.04$ & 0.048 \\
\hline TG, mmol/l & $1.08 \pm 1.15$ & $1.00 \pm 0.64$ & 0.726 \\
\hline TC, $\mathrm{mmol} / \mathrm{l}$ & $3.57 \pm 0.97$ & $3.73 \pm 0.59$ & 0.445 \\
\hline $\mathrm{HDL}, \mathrm{mmol} / \mathrm{I}$ & $1.09 \pm 0.28$ & $1.09 \pm 0.35$ & 0.980 \\
\hline LDL, mmol/I & $3.96 \pm 8.91$ & $2.40 \pm 0.64$ & 0.343 \\
\hline Amylase, U/L & $53.81 \pm 18.71$ & $60.37 \pm 37.57$ & 0.396 \\
\hline Potassium, mmol/L & $8.20 \pm 24.28$ & $3.84 \pm 0.42$ & 0.329 \\
\hline Sodium, mmol/L & $136.28 \pm 7.16$ & $136.31 \pm 2.70$ & 0.987 \\
\hline Chlorine, $\mathrm{mmol} / \mathrm{L}$ & $96.60 \pm 18.03$ & $97.39 \pm 18.38$ & 0.867 \\
\hline Calcium, mmol/L & $2.02 \pm 0.11$ & $1.92 \pm 0.38$ & 0.205 \\
\hline PT, s & $12.66 \pm 1.18$ & $12.77 \pm 1.55$ & 0.765 \\
\hline $\mathrm{D}-\mathrm{D}, \mathrm{mg} / \mathrm{L}$ & $0.32 \pm 0.18$ & $0.77 \pm 1.72$ & 0.163 \\
\hline APTT, s & $29.90 \pm 3.04$ & $33.55 \pm 16.74$ & 0.245 \\
\hline Fibrinogen, g/L & $4.17 \pm 2.42$ & $3.36 \pm 0.78$ & 0.086 \\
\hline INR & $1.08 \pm 0.07$ & $1.11 \pm 0.14$ & 0.246 \\
\hline $\mathrm{CD} 3, / \mu \mathrm{L}$ & $590.75 \pm 214.04$ & $527.36 \pm 273.98$ & 0.541 \\
\hline $\mathrm{CD} 3+\mathrm{CD} 4, / \mu \mathrm{L}$ & $355.50 \pm 145.18$ & $291.82 \pm 167.42$ & 0.340 \\
\hline $\mathrm{CD} 3+\mathrm{CD} 8, / \mu \mathrm{L}$ & $180.25 \pm 109.32$ & $198.18 \pm 125.78$ & 0.718 \\
\hline CD3 + CD19, / $\mu \mathrm{L}$ & $109.00 \pm 100.59$ & $89.82 \pm 40.81$ & 0.562 \\
\hline $\mathrm{CD} 56+\mathrm{CD} 16+\mathrm{CD} 3, / \mu \mathrm{L}$ & $113.25 \pm 63.18$ & $98.82 \pm 77.85$ & 0.629 \\
\hline \multicolumn{4}{|c|}{$\begin{array}{l}\text { Abbreviations: WBC, white blood cell count; RBC, red blood cell count; HGB, hemoglobin; ESR, } \\
\text { erythrocyte sedimentation rate; HCT, hematocrit; PLT, Platelet count; LYMP, lymphocyte count; MON\#, } \\
\text { monocytes absolute count; NEUT, neutrophil count; EO\#, eosinophil absolute count; CRP, C-reactive } \\
\text { protein; SAA, serum amyloid A; CT, calcitonin; TB, Total bilirubin; D-BIL, direct bilirubin; ALT, alanine } \\
\text { aminotransferase; AST, aspartate aminotransferase; AKP, alkaline phosphatase; LDH, Lactate } \\
\text { dehydrogenase; CK, creatine kinase; TG, triglyceride; TC, total cholesterol; HDL, high-density lipoprotein; } \\
\text { LDL, low-density lipoprotein; PT, prothrombin time; D-D, D dimer; APTT, activated partial thromboplastin } \\
\text { time; INR, international normalized ratio; CD, cluster differentiation antigen; C, complement. }\end{array}$} \\
\hline
\end{tabular}


Abbreviations: WBC, white blood cell count; RBC, red blood cell count; $H G B$, hemoglobin; ESR, erythrocyte sedimentation rate; $\mathrm{HCT}$, hematocrit; PLT, Platelet count; LYMP, lymphocyte count; MON\#, monocytes absolute count; NEUT, neutrophil count; EO\#, eosinophil absolute count; CRP, C-reactive protein; SAA, serum amyloid A; CT, calcitonin; TB, Total bilirubin; D-BIL, direct bilirubin; ALT, alanine aminotransferase; AST, aspartate aminotransferase; AKP, alkaline phosphatase; LDH, Lactate dehydrogenase; $\mathrm{CK}$, creatine kinase; TG, triglyceride; TC, total cholesterol; HDL, high-density lipoprotein; LDL, low-density lipoprotein; PT, prothrombin time; D-D, D dimer; APTT, activated partial thromboplastin time; INR, international normalized ratio; $C D$, cluster differentiation antigen; $C$, complement.

\subsection{Evaluation of patients' mental health using HADS, the Van Dream Anxiety Scale, and the MMSE scale}

The mean \pm standard deviation of anxiety scores reflecting the mental health of patients based on the HADS and Van Dream Anxiety Scale (presented in Table 1).

Regarding the mental health aspects, a significant difference was noted among the anxiety scores of patients with severe infection and healthy controls $(P<0.001)$, besides the significant difference between patients with moderate infection and healthy controls shows $P=0.004$. The depression scores of patients with moderate infection differed significantly from those of healthy controls $(P<0.001)$. Analysis of the MMSE scores revealed that COVID-19 affects both patients with moderate infection and patients with severe infection.

As the patient's condition worsened, their anxiety score, depression score, and dream anxiety score increased (Fig. 3), but the MMSE score gradually decreased (Fig. 5). Figure 4 shows the positive ratio of HADS scores in healthy controls, patients with moderate infection, and patients with severe infection. We classified the HADS scores greater than 8 as abnormal, that is, anxiety or depression was positive. An obvious difference was noted in the positive rate among healthy controls, and patients with moderate or severe infection.

\subsection{Correlation and Mean Scores of Anxiety, Depression, and Dream Anxiety}

Figure 5 shows a linear plot of the dream anxiety scores, anxiety, and depression scores for healthy controls, patients with moderate infection, and patients with severe infection. Based on the absolute value of $\mathrm{R}$, no correlation was evident if values ranged from $0-0.09 ; 0.1-0.3$ indicated a weak correlation; $0.3-0.5$ indicated a moderate correlation; and $0.5-1.0$ indicated a strong correlation. We concluded that in patients with moderate infection, the depression scores had a moderately positive correlation with dream anxiety scores $(R=0.313, P<0.001)$. In patients with severe infection, the anxiety score was positively correlated with the dream anxiety score $(R=0.330, P<0.001)$, and the depression score was moderately and positively correlated with the dream anxiety score $(R=0.385, P<0.001)$. Although the anxiety scores and depression 
scores of the patients with moderate infection and those with severe infection were strongly positively correlated, no significant differences were noted between the two groups $P>0.05$. Furthermore, no correlations were noted between the number of lung lesions and these scores.

\section{Discussion}

Although the nucleic acid sequence and gene characteristics of SARS-CoV-2 differ from those of SARS coronavirus and MERS coronavirus, they are similar to some beta coronaviruses from bats and have a homology of more than $85 \%$ with BAT-sI-covzc $45 .{ }^{3}$ When the virus is isolated from patients' lung fluid, blood, and nasal polyp sub-samples, it has shown typical coronavirus characteristics under electron microscopy. Furthermore, its transformation into SARS-CoV-2 is a characteristic of the new beta genera, which includes features such as capsules, round or oval particles that are often polymorphic, and 60$140 \mathrm{~nm}$ in diameter. ${ }^{3}$

In a recent study, SARS-CoV-2 and SARS-CoV were shown to share the angiotensin-converting enzyme 2 (ACE2) receptor. ${ }^{14}$ Furthermore, tissues with ACE2 expression may become target organs of SARS-CoV-2. ${ }^{14}$ The spike glycoprotein (S protein) on the surface of SARS-CoV mediates receptor recognition and membrane fusion. ${ }^{15}$ The $\mathrm{S} 1$ subunit contains the binding domain (RBD) of the receptor that binds directly to ACE2. ${ }^{16}$

Moreover, SARS-CoV-2 has the same S protein as SARS; thus, SARS-CoV-2 may use ACE2 to infect. ${ }^{17}$ Surprisingly, a recent bioRxiv preprint shows that the affinity between ACE2 and the RBD of SARS-CoV-2 is 10-20 times higher than that between ACE2 and the RBD of SARS-CoV. ${ }^{18}$ Therefore, the S protein largely determines host specificity and infectivity of the new coronavirus, and is also a key target for the development of vaccines and therapeutic antibodies, and clinical diagnosis.

Among the first deaths in mainland China due to COVID-19, Xu et al. found that acute respiratory distress syndrome (ARDS) in patients with severe infection might be due to SARS-CoV-2 and subsequent interstitial lung disease. This finding created increased understanding of the disease. Moderate microvascular steatosis in liver specimens might explain the reduced levels of AST observed among the patients with moderate to severe disease. At the same time, Xu et al. believed that lymphopenia was a common feature of patients with COVID-19, and this might be related to disease severity and mortality. ${ }^{19}$

In addition to droplet infection, many people are concerned about the possibility of vertical transmission. However, according to Chen $\mathrm{H}$, et al., nine cases of COVID-19 have been treated by Wuhan University Zhongnan Hospital from January 20, 2020 to January 31, 2020. A retrospective study has been conducted, based on the medical records of pregnant women with pneumonia and the results of tests on amniotic fluid, umbilical cord blood, breast milk, and neonatal throat swab samples. They concluded that there is currently no evidence that SARS-CoV-2 may cause fetal infections caused by vertical transmission in the womb. ${ }^{20}$ Moreover, previous studies of SARS-CoV showed no evidence of SARS infection during the perinatal period. ${ }^{21}$ 
Currently, exploration of the important prognostic factors and therapeutic drugs for COVID-19 is a matter of urgent concern. On February 10, 2020, after comparing the clinical characteristics, imaging characteristics, and treatment methods of patients with mild, moderate, and severe infections, an expert team at the Beijing Ditan Hospital concluded that the neutrophil-to-lymphocyte ratio (NLR) may be an influential factor in the prognosis and early identification of patients with severe infection. Patients over 50 years of age with an NLR $\geq 3.13$ constitute a high-risk group prone to severe infections and should be admitted to the ICU immediately, if necessary. ${ }^{22}$

Previously, Holshue et al. ${ }^{23}$ found that remdesivir was an effective drug used during the diagnosis and treatment of the first COVID-19 patient in the United States. Although remdesivir has not been licensed or approved for use in mainland China, a phase II clinical study of the drug, confirms that long-term use of remdesivir is safe. ${ }^{24}$

As a designated hospital for the treatment of patients with severe COVID-19 infection in the Jiangxi Province, our hospital has had a concentration of most of the severe cases within the province. Patients with severe infection have received effective symptomatic treatment and respiratory support (such as invasive ventilation or extracorporeal membrane oxygenation).

The modern medical model is a bio-psycho-social model, and anxiety and depression are the most common types of emotional disorders that may occur and pose a significant public health burden. The human-tohuman transmission characteristics, extreme discomfort caused by critical illness, desperation caused by strict isolation precautions within the hospital environment, and uncertainty of the prognosis can all affect patients with severe infection. The psychological burden can lead to abnormal mentation. ${ }^{25}$

Physiological suffering accompanied by anxiety and depression can negatively affect the quality of life and prognosis of the patient. Treatment of physical diseases alone cannot relieve anxiety and depression. Therefore, in addition to treating physical diseases, doctors need to actively pay attention to the treatment of anxiety and depression. Research into the psychology of patients infected with the new coronavirus pneumonia (especially as it relates to symptoms of anxiety and depression) and targeted psychological interventions to alleviate the patient's psychological burden will likely provide novel ideas for clinical treatment and may be of practical significance.

\section{Limitations}

This study was affected by the following limitations: only 60 patients were included in the study (30 patients with moderate infection, 21 patients with severe infection, and nine critical patients). A large number of confirmed patients were continuously being admitted to the hospital during analysis of the data. Therefore, the researchers only obtained data from patients diagnosed with the infection in some laboratories within Jiangxi Province during the study period.

Secondly, because the patients were selected specifically from Jiangxi Province, which included five cities, the results obtained were from a single study center. Furthermore, most patients were not discharged when 
the analysis of the data was completed. Thus, it was not possible to estimate mortality and cure rates, among other factors.

\section{Conclusion}

The history of exposure and abnormalities on chest CT scans showed considerable diagnostic value. Patients with severe infection had more pain and sputum in the throat area than patients with moderate infection. Patients with blood type A might be more susceptible to COVID-19, and lymphopenia indicated worsening of COVID-19. In patients with moderate infection and those with severe infection, the depression score and dream anxiety score were moderately and positively correlated. In patients with severe infection, the anxiety score and dream anxiety score were also moderately and positively correlated.

\section{List Of Abbreviations}




\begin{tabular}{|ll|}
\hline Abbreviations & Full name \\
\hline COVID-19 & coronavirus disease-19 \\
\hline SAA & serum amyloid A \\
\hline WHO & World Health Organization \\
\hline SARS-CoV & severe acute respiratory syndrome coronavirus \\
\hline 2019-nCoV & 2019-New Coronavirus \\
\hline SARS-CoV-2 & acute respiratory syndrome coronavirus 2 \\
\hline MERS-CoV & Middle Eastern respiratory syndrome coronavirus \\
\hline PHEIC & Public Health Emergency of International Concern \\
\hline HADS & Hospital Anxiety and Depression Scale \\
\hline MMSE & Mini-Mental State Examination \\
\hline CT & computed tomography \\
\hline RT-PCR & real-time fluorescent reverse transcription polymerase chain reaction \\
\hline PaO 2 & partial arterial pressure of oxygen \\
\hline ICU & intensive care unit \\
\hline BMI & body mass index \\
\hline SAA & serum amyloid A \\
\hline AST & aspartate aminotransferase \\
\hline LDL & low-density lipoprotein \\
\hline ACE2 & angiotensin-converting enzyme 2 \\
\hline RBD & acute respiratory distress syndrome \\
\hline ARDS & neutrophil-to-lymphocyte ratio \\
\hline NLR & \\
\hline
\end{tabular}

\section{Declarations}

\section{Ethical approval and consent to participate}

The study methods and protocols were approved by the Medical Ethics Committee of the First Affiliated Hospital of Nanchang University (Nanchang, China) and followed the principles of the Declaration of Helsinki. All subjects were notified of the objectives and content of the study and latent risks, and then provided written informed consent to participate. 


\section{Patient consent for publication}

Not applicable.

\section{Availability of data and materials}

The datasets used and/or analyzed during the present study are available from the corresponding author on reasonable request.

\section{Funding}

Emergency Science and Technology Project for COVID-19 of Jiangxi Province (202011-2).

\section{Competing interests}

This study did not receive any industrial support. The authors have no competing interests to declare regarding this study

\section{Authors' contributions}

$\mathrm{CYZ}, \mathrm{WZ}$, and JZ conceived and designed the present study. XPL, FX and YS were responsible for acquiring the data, designing the figures and tables and drafting the manuscript. MHZ, JJY contributed to the acquisition of the data and interpreting the results. FL assisted in the acquisition of the data and drafting the manuscript. WZ, SGX assisted in the acquisition and analysis of the data with constructive discussion.

\section{Acknowledgements}

Not applicable.

\section{References}

1. National Health Commission of the People' s Republic of China. Latest developments in epidemic control on Feb 24 (1). http://en.nhc.gov.cn/2020-02/24/c_76853.htm. (accessed Feb 24, 2020).

2. XINHUANET News Report. (http://www.xinhuanet.com/english/2020-02/13/c_138779875.htm).

3. Sanz F, Gimeno C, Lloret T, et al., Relationship between the presence of hypoxemia and the inflammatory response measured by $\mathrm{C}$-reactive protein in bacteremic pneumococcal pneumonia. Eur Respir, 2011. 38 (suppl 55): 2492.

4. XINHUANET News Report. (http://www.xinhuanet.com/english/2020-01/09/c_138690570.htm).

5. SU S, WONG G, SHI W FF, LIU J, LAI A CK, ZHOU J Y, et al. Epidemiology, genetic recombination, and pathogenesis of coronaviruses. Trends Microbiol, 2016, 2(6):490-502.

6. CUI J, LI F, SHI ZL. Origin and evolution of pathogenic coronaviruses[J]. Nat Rev Microbiol, 2019, 17:181-92.

7. National Health Commission of the People's Republic of China. Mental health services deployed across China in fight against epidemic. Handbook of Prevention and Treatment of the Pneumonia 
Caused by the Novel Coronavirus (2019-nCoV). http://en.nhc.gov.cn/2020-02/06/c_76295.htm. (accessed Feb 6, 2020; chindaily.com.cn).

8. Zhou P, Yang X, Wang X, et al. Discovery of a novel coronavirus associated with the recent pneumonia outbreak in humans and its potential bat origin. bioRxiv, 2020:2020-2021.

9. Information Office of the State Council. The Information Office held a press conference on the joint prevention and control of pneumonia epidemic of new coronavirus infection (2020-01-26) [EB / OL]. Http://www.scio.gov.cn / xwfbh / xwbfbh / wqfbh / 42311/42478 / index.htm.

10. Chan JF, Yuan S, Kok KH, et al. A familial cluster of pneumonia associated with the 2019 novel coronavirus indicating person-to-person transmission: a study of a family cluster. Lancet. 2020 Jan 24. pi: S0140-6736(20)30154-9.

11. N. Chen et al., Epidemiological and clinical characteristics of 99 cases of 2019 novel coronavirus pneumonia in Wuhan, China: a descriptive study. Lancet, (2020).

12. National Health Commission of the People's Republic of China. Commonalities in cured COVID-19 cases. http://en.nhc.gov.cn/2020-02/24/___76883.htm. (accessed Feb 24, 2020).

13. National Health Commission of the People's Republic of China. Mental health services deployed across China in fight against epidemic. http://en.nhc.gov.cn/2020-02/13/c_76546.htm. (accessed Feb 13, 2020).

14. R. Cho et al., Dorsal diaphragmatic excursion tracks transpulmonary pressure in ventilated ARDS patients: a potential non-invasive indicator of lung recruitment? Crit Care, 2016. 20(Suppl 2).

15. Simmons G, Zmora P, Gierer S, Heurich A, PohImann S. Proteolytic activation of the SARS-coronavirus spike protein: cutting enzymes at the cutting edge of antiviral research. Antiviral Res. 2013;100(3):60514.

16. Li F, Li W, Farzan M, Harrison SC. Structure of SARS coronavirus spike receptor binding domain complexed with receptor. Science. 2005;309(5742):1864-8.

17. Hoffmann M, Kleine-Weber H, Krüger N, Müller M, Drosten C, Pöhlmann S. The novel coronavirus 2019 (2019-nCoV) uses the SARS-coronavirus receptor ACE2 and the cellular protease TMPRSS2 for entry into target cells. bioRxiv. 2020.

18. Jason S. McLellan et al. Cryo-EM Structure of the 2019-nCoV Spike in the Prefusion Conformation. bioRxiv. 2020.

19. Zhe Xu et al. Pathological findings of COVID-19 associated with acute respiratory distress syndrome. The Lancet, 2020.

20. Chen $\mathrm{H}$, Guo J, Wang $\mathrm{C}$, et al. Clinical characteristics and intrauterine vertical transmission potential of COVID-19 infection in nine pregnant women: a retrospective review of medical records. Lancet 2020; published online Feb 12.

21. Wong SF, Chow KM, Leung TN, et al. Pregnancy and perinatal outcomes of women with severe acute respiratory syndrome. Am J Obstet Gynecol 2004; 191: 292-97.

22. Neutrophil-to-Lymphocyte Ratio Predicts Severe IIIness Patients with 2019 Novel Coronavirus in the Early Stage. medRvix, Posted February 12, 2020. 
23. Michelle L. Holshue, et al. First case of 2019 novel coronavirus in the united states, NEJM, 2020.

24. Sabue Mulangu, et al. A randomized, controlled trail of Ebola virus disease therapeutics. N Engl J Med. 381(24),2293-2303.

25. Leung CW, Kwan YW, Ko PW, Chiu SS, Loung PY, Fong NC, Lee LP, Hui YW, Law HK, Wong WH, Chan KH, Peiris JS, Lim WW, Lau YL, Chiu MC. Severe acute respiratory syndrome among children. Pediatrics. 2004, 113(6):e535-43.

\section{Figures}




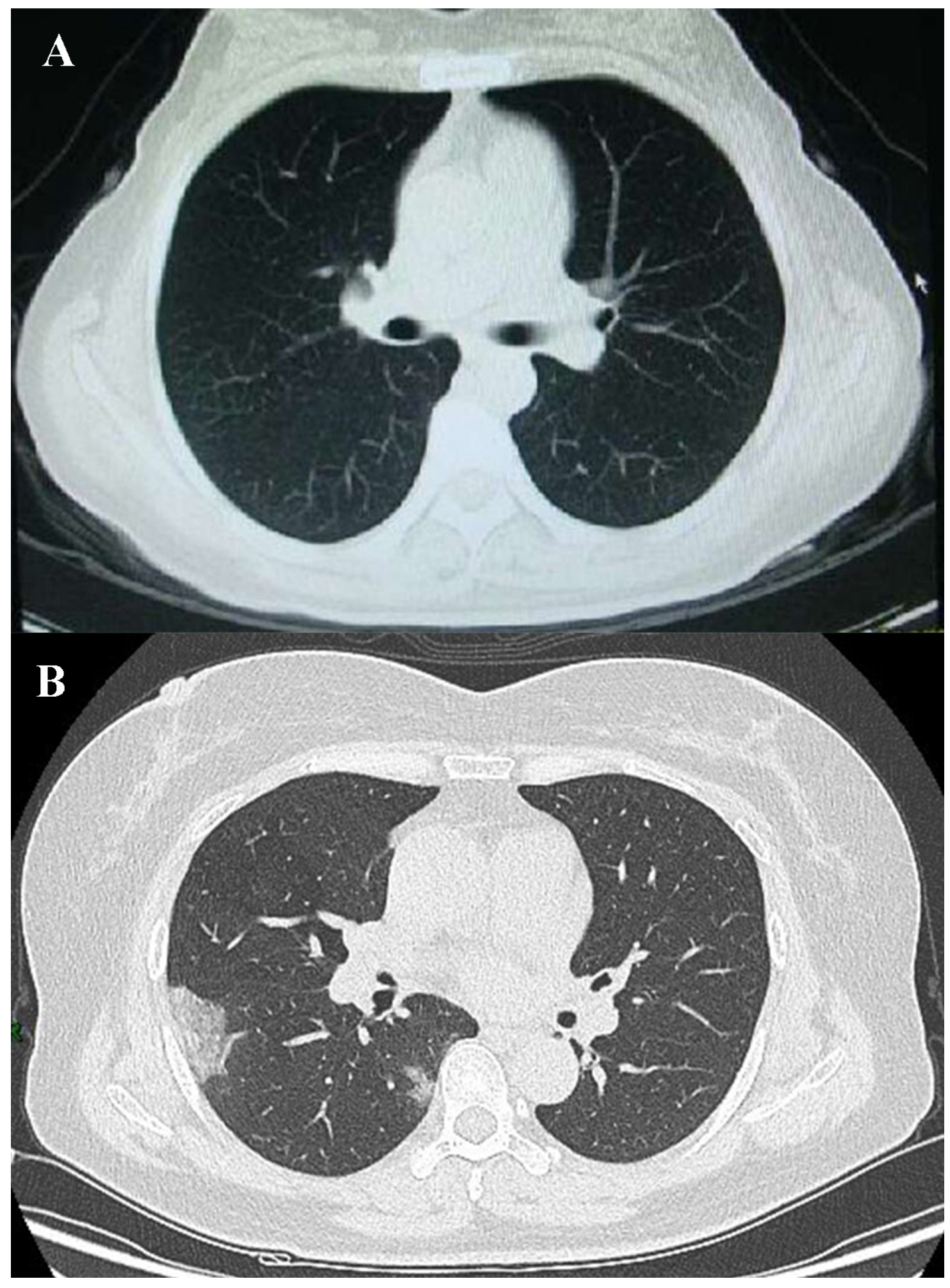

Figure 1

CT imaging of lungs in healthy controls and patient with COVID-19. Notes: Figure 1.A The healthy person has no lesions in the lungs; Figure 1.B The average number of lesions in the lungs of patients with COVID19 is $2.68 \pm 1.61$. 


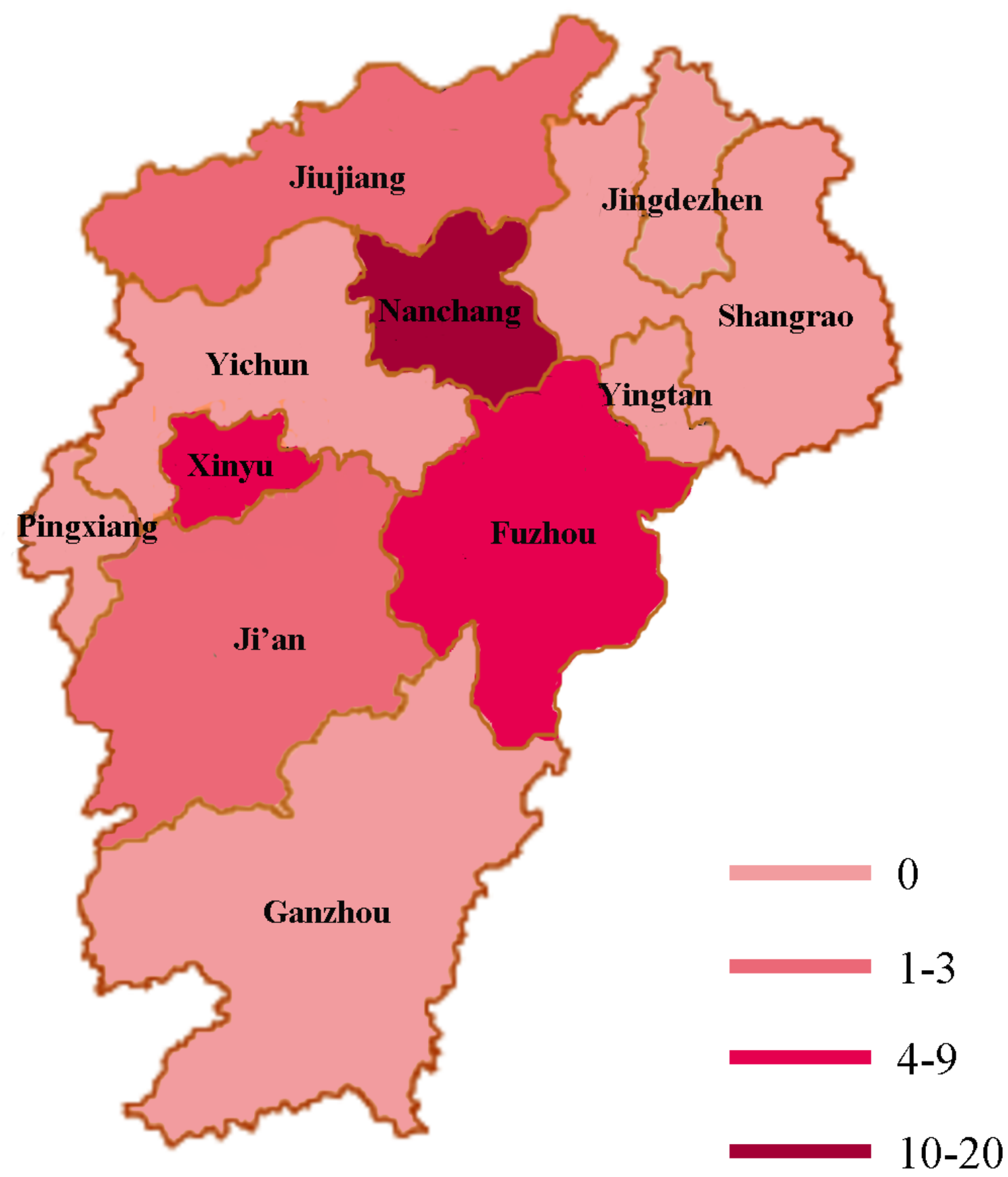

\section{Figure 2}

Distribution of severe and critical patients. Notes: Of the 30 severe patients (including 21 severe and 9 critical patients), 19 were from Nanchang, 4 were from Fuzhou, 4 were from Xinyu, 2 were from Jiujiang, and 1 was from Ji'an. Note: The designations employed and the presentation of the material on this map do not imply the expression of any opinion whatsoever on the part of Research Square concerning the legal status of any country, territory, city or area or of its authorities, or concerning the delimitation of its frontiers or boundaries. This map has been provided by the authors. 


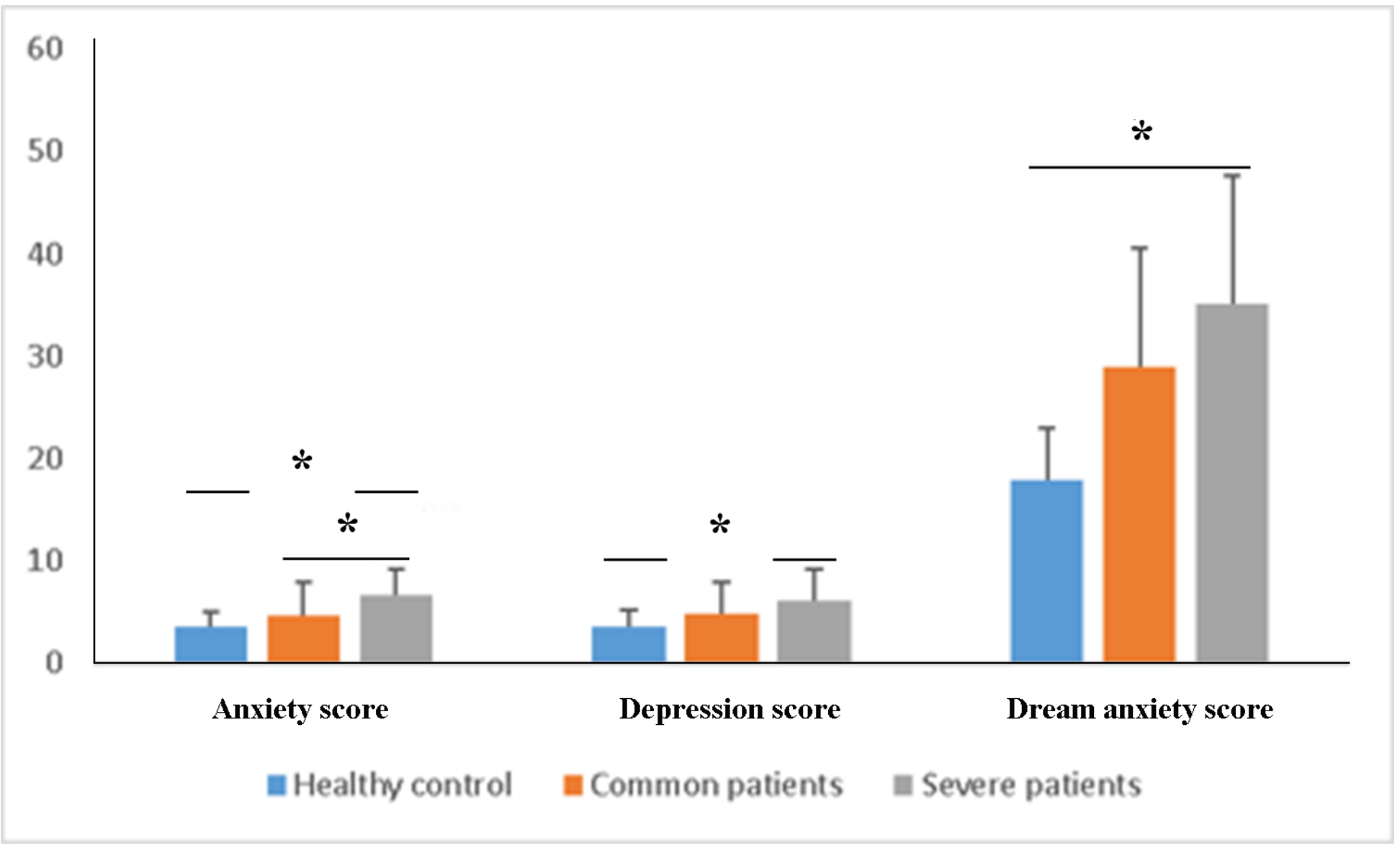

\section{Figure 3}

Mental health assessments for Health controls, common and severe patients. Notes: Anxiety scores and depression scores were obtained from HADS, and dream anxiety scores were obtained from the Van Dream Anxiety Scale. * indicates that the difference between the two groups is statistically significant. Abbreviations: HADS, the Hospital Anxiety and Depression Scale. 
A

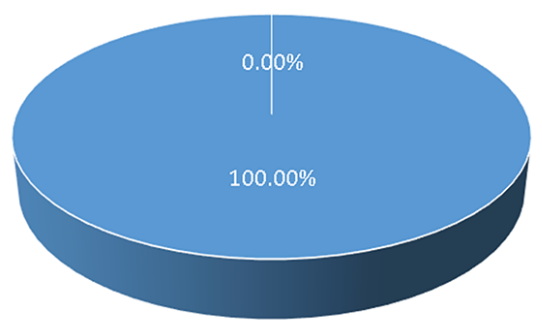

- Normal = Abnormal

D

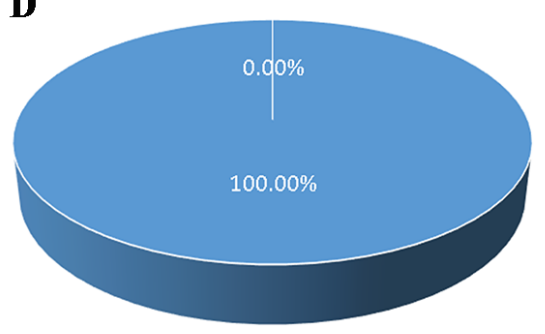

ఐ Normal $₫$ Abnormal
B

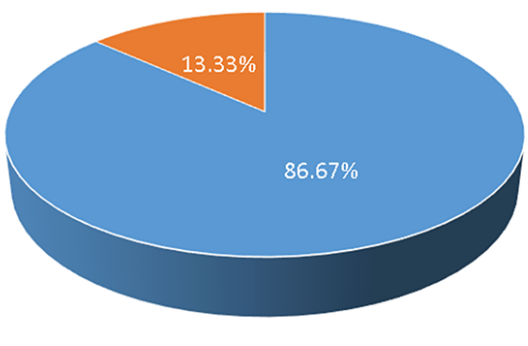

- Normal - Abnormal

$\mathbf{E}$

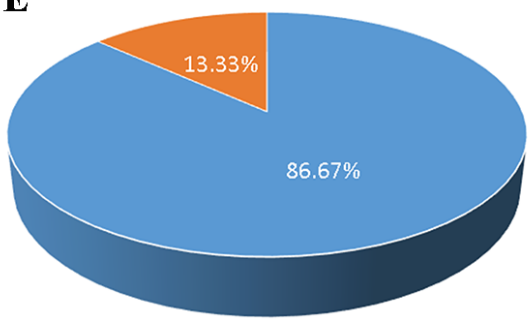

- Normal - Abnormal
C

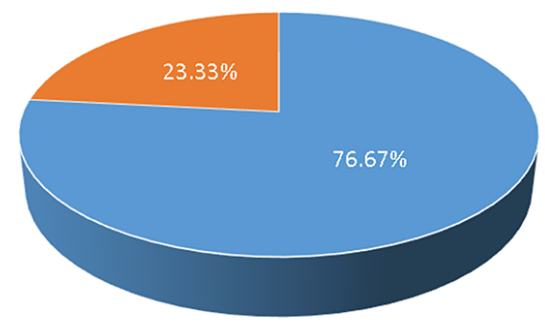

- Normal | Abnormal

$\mathbf{F}$

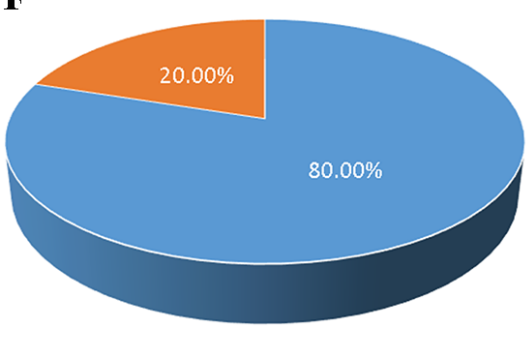

- Normal - Abnormal

Figure 4

Positive ratio of HADS scores in healthy controls, common patients, and severe patients. Notes: A: Positive ratio of anxiety scores in healthy controls. B: Positive ratio of anxiety scores in common patients. C: Positive ratio of anxiety scores in severe patients. D: Positive ratio of depression scores in healthy controls. E: Positive ratio of depression scores in common patients. F: Positive ratio of depression scores in severe patients. HADS score greater than 8 is classified as abnormal, that is, anxiety or depression is positive. Abbreviation: HADS, the Hospital Anxiety and Depression Scale. 


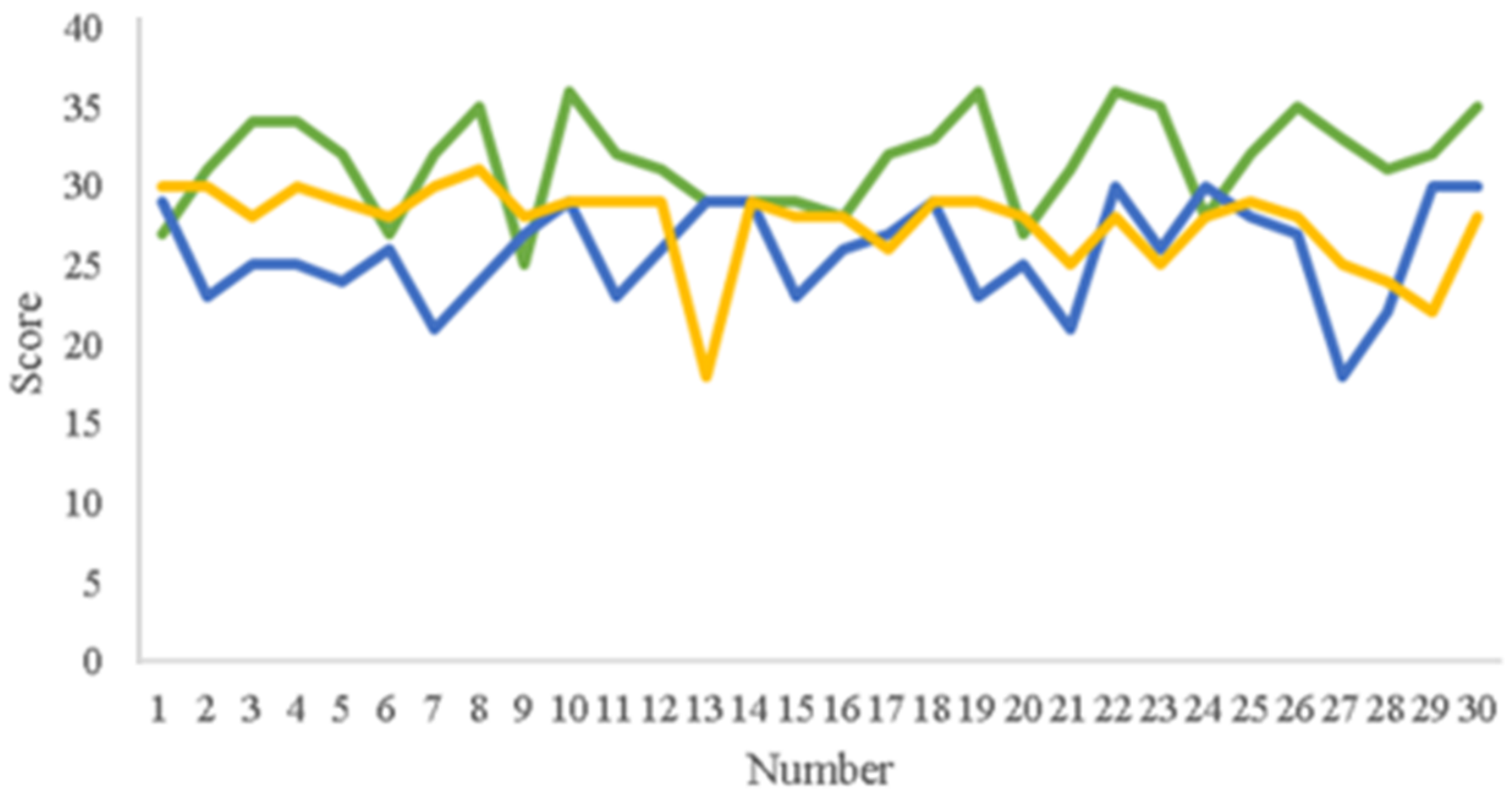

$\longrightarrow$ Healthy control $\longrightarrow$ Common patients $\longrightarrow$ Severe patients

\section{Figure 5}

Comparison of MMSE scores between healthy controls, common patients, and severe patients. Notes: As the patient's condition worsens, the patient's MMSE score gradually decreases. 

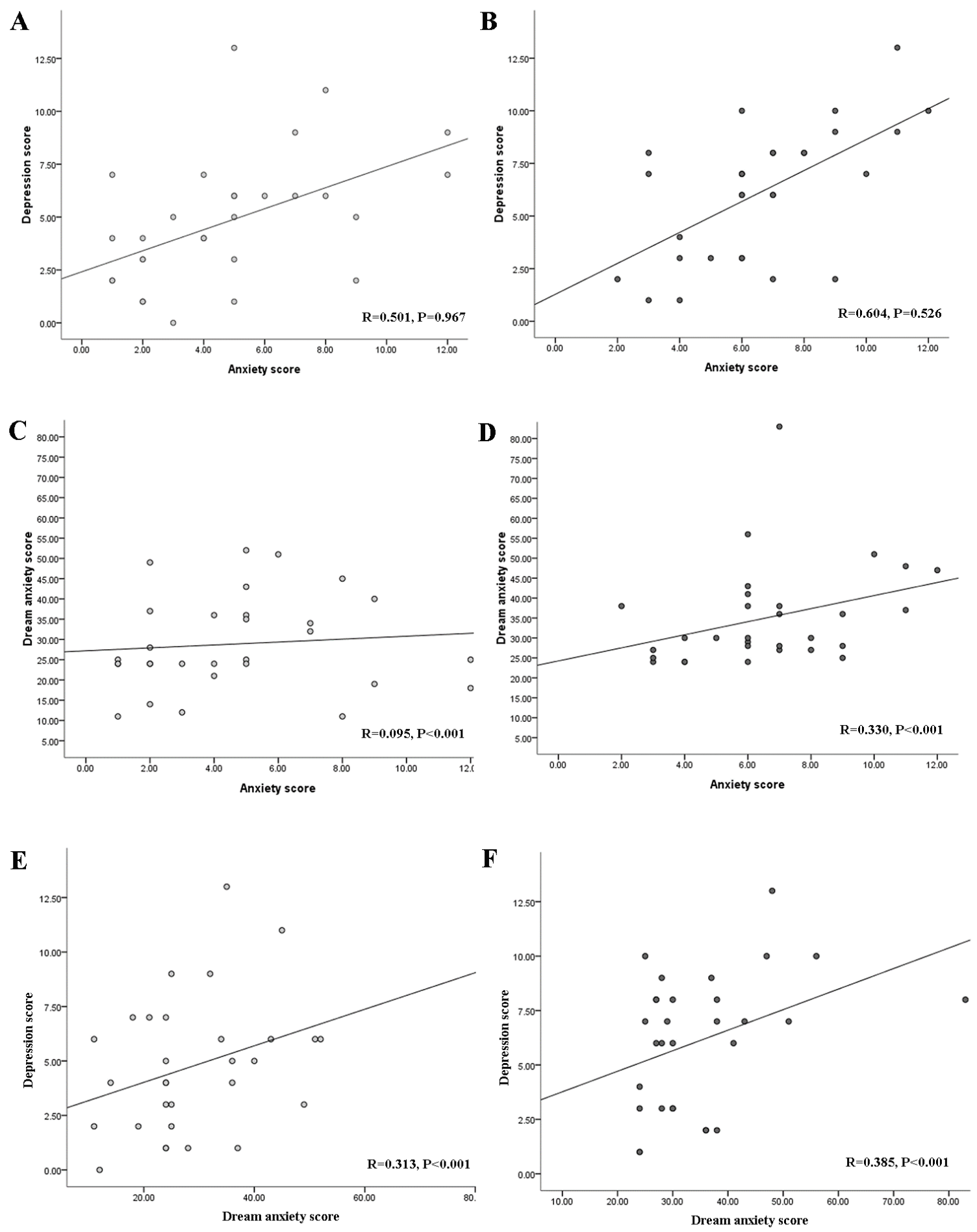

\section{Figure 6}

Linear plots in dream anxiety scores, anxiety and depression scores of healthy controls, common patients, and severe patients. Notes: A: A linear plot of anxiety and depression scores in common patients with COVID-19. B: A linear plot of anxiety and depression scores in severe patients with COVID-19. C: A linear plot of anxiety and dream anxiety scores in common patients with COVID-19. D: A linear plot of anxiety and dream anxiety scores in severe patients with COVID-19. E: A linear plot of dream anxiety and depression scores in common patients with COVID-19. F: A linear plot of dream anxiety and depression scores in severe 
patients with COVID-19 There was no correlation between lesions and these scores. Abbreviation: COVID-19, coronavirus disease-19.

\section{Supplementary Files}

This is a list of supplementary files associated with this preprint. Click to download.

- S3table.docx

- S1table.docx

- S2table.docx 